\title{
Cómo eleVAR LA RENTABILIDAD DE UNA EMPRESA
}

\section{APLICACIÓN DE UN ERP NACIONAL}

\section{Luis Espinal Rojas}

\section{Resumen}

Este artículo intenta dar a conocer las ventajas que obtendría una empresa si aplicara tecnologías de la información (TI) como herramientas estratégicas para alcanzar sus objetivos. Para el estudio se elegió una empresa con varios problemas, principalmente en la gestión del inventario, debido a una deficiente administración en la cadena de suministros. Se buscó solucionar los problemas aplicando herramientas de las TI para demostrar cómo estas pueden mejorar notablemente la gestión de sus suministros, con el objetivo principal de elevar la rentabilidad y optimizar la imagen de la empresa a corto plazo. La meta era incrementar sus niveles de venta en un 10\%, pues por la deficiencia en sus procesos, había descendido a cifras que debían elevarse.

Los resultados de la implementación se dieron en el plazo esperado, con un incremento en la venta de los productos de 21,66 \% en el 2013 con respecto al año anterior, lo que permitió que el nivel de inventario se redujera en un 22,5\%, lo que da como resultado una reducción en los costos de inventario, operaciones, logística y compras. Otro tema para resaltar es el grado de satisfacción de los clientes, pues al manejar de manera adecuada los inventarios, estos encontraron lo que necesitaban en el momento adecuado y al precio justo.

Palabras clave: Modelamiento de procesos/gestión de inventario perpetuo/metodologías ágiles / sistema de información / ERP / arquitectura de software / gestión de cadena de suministros / nteligencia de negocios / dinámica de sistemas 


\section{Introducción}

Toda empresa, sea pequeña, mediana o grande necesita herramientas de tecnologías de información, tanto así que se pueden encontrar empresas que promueven el desarrollo de software exclusivamente para medianas y pequeñas empresas y lo definen explícitamente: "[.. .] no es un ERP de empresas grandes que fue recortado para dárselo a las pymes, sino que fue creado específicamente para ellas" (Valencia 2004) para poder tener ventaja competitiva frente a sus pares. Así, como primer paso para la implementación del sistema de información, se debe establecer el planeamiento estratégico de la empresa, sus principales actividades, sus objetivos y metas, y tener conocimientos de cuáles son los procesos críticos para modelar.

El modelamiento de los procesos de la empresa y el desarrollo del sistema de información ofrecen mejoras que se pueden aplicar a los procesos actuales; se puede desarrollar un sistema de pronóstico de demanda para apoyar el área de ventas, pues se trata de que tanto esta como la de inventario estén sincronizadas. Es claro que el desarrollo de estos sistemas no es de bajo costo, el tiempo que utiliza es amplio y el retorno de la inversión no se aprecia a corto plazo; por ello muchas empresas no confían o tienen ideas erróneas de un sistema de información que les hace pensar que estas herramientas no son necesarias. Para evitar estos inconvenientes se deben tener bien definidos los objetivos de la empresa y sus procesos, y demostrar que estos necesitan soporte de herramientas de tecnología de información que le generen un valor agregado a la empresa y mejoren su rentabilidad.

Las herramientas de TI juegan un papel importante en esta solución; los cambios de paradigmas y la alta competitividad que existe hacen que ya no solo se necesite un ERP tradicional que nos proporcione información, sino que también nos brinde información resumida entendible por la mayoría de los usuarios (Zhou 2012). Estos dos sistemas se han relacionado y van juntos de la mano, inteligencia de negocios y sistemas de información. Esto facilita la toma de las decisiones más importantes para la empresa; se mejora su rentabilidad y se le da mejor uso a la información. Para alcanzar el éxito en el proyecto, se tienen que analizar los componentes de la gestión de la cadena de suministros (SCM). Los flujos principales en los que se enfocará son el inventario, el flujo de información y el financiero (Othman y Mustaffa 2012).

La solución propuesta debe ser modelada para luego ser simulada y así comprobar el impacto de esta en el negocio; existen muchas herramientas de simulación, como Arena, PowerSim, Matlab, @Risk, con los que se podría demostrar el cambio al implementar una buena gestión de la cadena de suministro. Las ventajas se pueden observar mediante indicadores y tableros de comando, los 
cuales apoyan en el monitoreo de las áreas involucradas. La principal ventaja que ofrece un ERP es precisamente el tener todo integrado en una misma base de datos (Sánchez 2007) y obtener así herramientas suficientes para desarrollar un planeamiento de recursos e implementar un sistema de inteligencia de negocios: bussiness intelligence (BI)

\section{Descripción del problema}

\subsection{Realidad del problema}

El mercado de los metales no ferrosos es muy cambiante, es por esto que se deben monitorear constantemente los diversos factores que influyen en la variación del precio de dichos metales; se pueden encontrar variables tanto del micro como del macro entorno; algunas de ellas pueden ser políticas, naturales, sociales, y especulaciones del mercado. Por ese motivo las empresas que trabajen con estos recursos deben estar al tanto de sus inventarios, pues un cambio drástico del precio puede afectar la rentabilidad de sus operaciones. En la figura 1 se puede apreciar la variación de los metales en el tiempo.

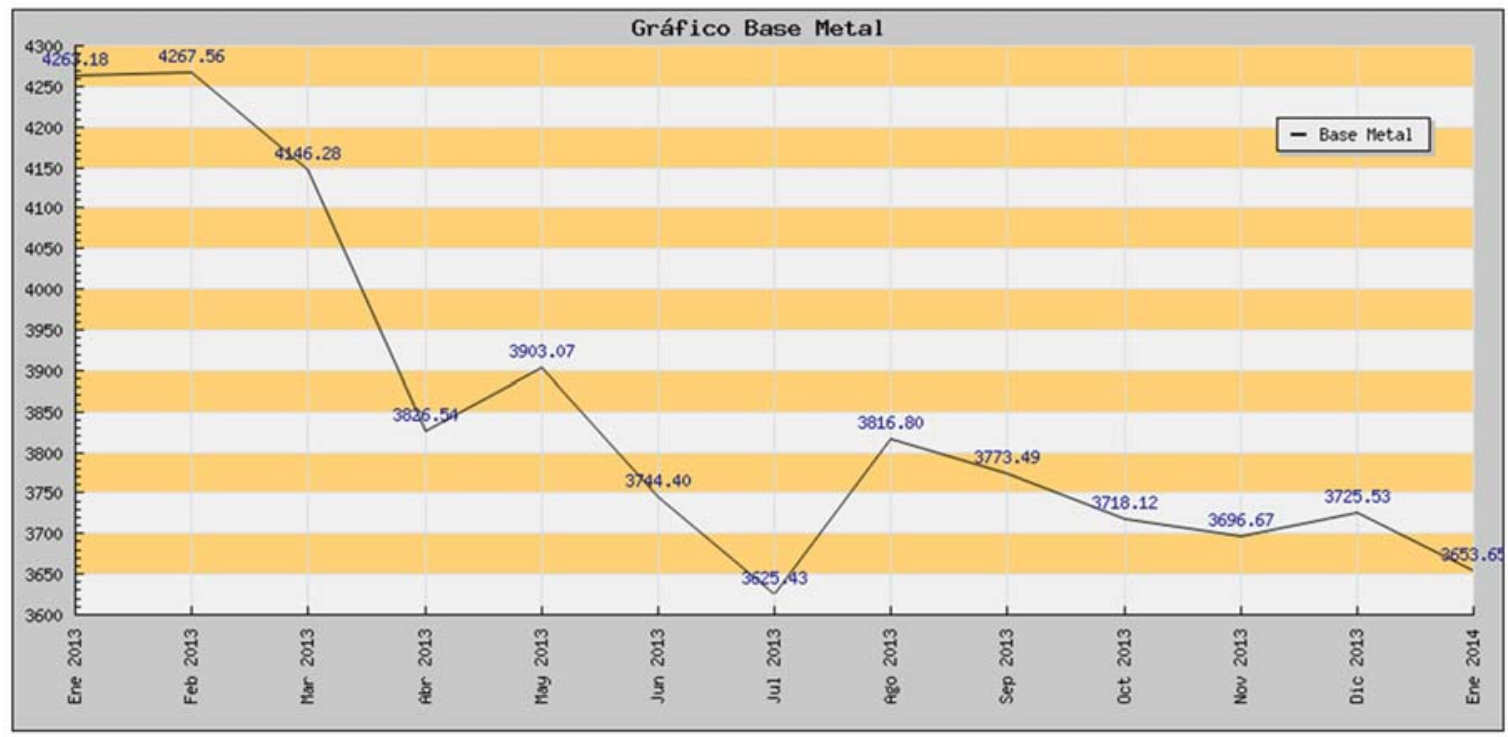

Figura 1. Variación del precio del latón

Elaboración propia. 
La empresa escogida es una empresa peruana que se dedica a la venta de metales no ferrosos, como cobre, latón, al paca y zinc, y a la fabricación, reparación, distribución de instrumentos musicales de viento, los cuales usan como materia prima el latón.

\subsection{Definición del problema}

A continuación se enumeran los problemas más notorios de esta empresa:

a) La empresa carece de un esquema visible de todos sus procesos, por ello la trazabilidad y el manejo de materiales es deficiente; por lo tanto se requiere modelar todos sus procesos con el objetivo de mejorarlos, pues se necesita tener una gestión eficiente y eficaz del manejo del inventario y la producción para satisfacer la demanda de los clientes.

b) No se dispone de un modelo de gestión de cadena de suministros.

c) Falta de comunicación o relación con los proveedores o miembros de la cadena de suministros.

d) El inventario de materia prima y del producto terminado presenta una gestión deficiente.

e) Incumplimiento de las fechas de entrega de los pedidos.

f) Los roles y responsabilidades del personal involucrado no se encuentran bien definidos.

g) No se cuenta con información suficiente para apoyar la toma de decisiones ni para gestionar el pronóstico de la demanda, para conocer cuánto de la materia prima se destina a la venta y cuánto a la producción.

\subsection{Objetivos}

El objetivo principal es proponer un modelo de gestión de cadena de suministros e implementar un sistema de información que permita mejorar la gestión del inventario de la empresa, teniendo un control de inventario perpetuo, cumpliendo las fechas de entrega y mejorando la relación con los clientes usando herramientas libres de la ingeniería de software. Los objetivos específicos que plantea el proyecto son:

a) Elevar el nivel de ventas actual a corto plazo en un $15 \%$, superando el ingreso actual de US\$1.500.000,00.

b) Realizar un modelamiento de los procesos de la empresa a fin de obtener su mejora.

c) Proponer un modelo de gestión de cadena de suministros para la empresa. 
d) Diseñar una arquitectura de sistema de software para la empresa.

e) Implementar un prototipo del sistema de información para la empresa elegida con el uso de metodologías ágiles.

f) Implementar una solución para la gestión de toma de decisiones por medio de herramientas de inteligencia de negocios (BI).

\section{Marco teórico}

\subsection{Enterprise resource planning (ERP)}

Un ERP (enterprise resource planning) es un sistema de gestión empresarial íntegro orientado a automatizar los procesos en la empresa, facilitar la planificación de todos los recursos de la empresa y mantener un control en tiempo real de todas las áreas involucradas.

La situación actual del sistema de información tradicional es adaptarse o desaparecer, hoy no basta con tener un programa funcionando de manera correcta en un ordenador, que tenga alta disponibilidad y que sea confiable, sino también que tenga portabilidad e información en tiempo real desde cualquier lugar. Las dificultades que se presentan al optar por un ERP están en el análisis de las necesidades de la empresa y la capacitación del personal para operar dicha herramienta.

\subsection{Arquitectura de software}

La arquitectura de software es la estructura de más alto de nivel de un sistema; se rige por los objetivos y restricciones que estas presentan. Los objetivos son escogidos de acuerdo con los requerimientos del sistema de información, que pueden ser funcionales o no funcionales, como la flexibilidad, el alcance, la interoperabilidad y la escalabilidad. Existen varias arquitecturas de software las cuales han ido evolucionando con el paso del tiempo, entre las que se pueden mencionar las siguientes:
a) monolítica;
b) cliente-servidor;
c) arquitectura por capas;
d) cliente servidor con capas; y
e) orientada a servicios. 


\subsection{Gestión de la cadena de suministros}

Todas las empresas buscan cumplir con todas las expectativas de los clientes, para lo que se necesita administrar la cadena de suministro, que es básicamente el proceso de planificación, ejecución y control de las operaciones con el fin de cumplir con los objetivos propuestos por la cadena. Estos objetivos son globales, es decir que involucran a todos los participantes de la cadena, tales como proveedor, productos, distribuidor, ventas y demás. El punto clave de la gestión es la distribución y el almacenaje de las existencias, que van desde el inicio de la cadena hasta llegar al usuario final, considerando todos los posibles escenarios que se puedan presentar en cada momento. Sus puntos críticos afectan directamente los siguientes procesos:

a) compras;

b) abastecimientos;

c) planificación;

d) producción;

e) almacenaje; $y$

f) distribución.

Y tiene los siguientes objetivos:

a) Configurar la cadena o red de distribución.

b) Identificar todos los participantes de la cadena.

c) Implementar una estrategia de distribución identificando las instalaciones y centros de distribución.

d) Juntar la información integrando los sistemas y el proceso de la cadena para desarrollar pronósticos a partir de información valiosa.

e) Administrar el manejo de inventario para satisfacer las necesidades del cliente en cuanto a cantidad, tiempo, calidad.

\subsection{Inteligencia de negocios}

Las empresas buscan siempre tomar las mejores decisiones para el cumplimiento de sus objetivos, una de las herramientas que las apoya es la inteligencia de negocios, pues utiliza información para facilitar la toma de decisiones. La recoge, la carga y la transforma (ETL), según los requerimientos o necesidades de los usuarios para luego almacenar esta información en un cubo de datos.

Un claro ejemplo de su utilización es la creación de reportes e indicadores (dashboard) para revisar el cumplimiento de los objetivos de la empresa. Por ejemplo, se quiere revisar cómo van las ventas de un determinado producto en cierto lugar y en un periodo de tiempo establecido; se cruzan los datos y se obtiene 
un reporte con el cual se pueden observar ciertos indicadores ya establecidos y así determinar si el producto tiene gran demanda o estacionalidad, para luego tomar decisiones como eliminar el producto o, quizás, manejar su demanda de acuerdo con los pronósticos. La inteligencia de negocios presenta muchos beneficios, los cuales se pueden describir en tres grandes principios:

a) reducción de costos;

b) mayor rentabilidad; $y$

c) satisfacción de los clientes.

\section{Estrategia de solución}

La empresa debe alinear sus procesos teniendo en cuenta las buenas prácticas, para eso el modelamiento de procesos es básico para comprender las actividades de la empresa, sus funciones, sus relaciones, sus interrelaciones y alcances, ya que muchas de ellas presentan cierto grado de complejidad; un modelamiento puede dar como resultado el mejoramiento de los procesos y su documentación.

La notación escogida será BPMN, principalmente porque es de fácil implementación, es entendible por usuarios no técnicos, otro factor es que abarca un enfoque más sistemático para el modelamiento de los procesos, involucra la parte tecnológica y administrativa, está alineada con el planeamiento estratégico del negocio, automatiza el funcionamiento de los procesos, permitiendo el control y monitoreo de las actividades; principalmente involucra negocio, procesos y gestión, lo que permite a una empresa estar más preparada para afrontar cambios en sus principales procesos.

El modelo de gestión de cadena de suministro tiene un horizonte de implementación largo, se pueden considerar años, y los cambios en ella a corto plazo presentan una gran inversión económica.

Para empezar, se debe tener en cuenta que está guiada por la estrategia global de la cadena, si bien es cierto que cada entidad involucrada tiene objetivos específicos como empresa, no se debe olvidar el objetivo principal de cadena.

Las estrategias de la cadena u objetivos es un proceso de cuatro pasos diseñado para satisfacer los cambios de la compañía y las diversas necesidades del cliente y los productos. Se describe cada uno de los pasos del proceso y da ejemplos de su publicación (Daniel Llamas y Delia Tasaico 2005). 


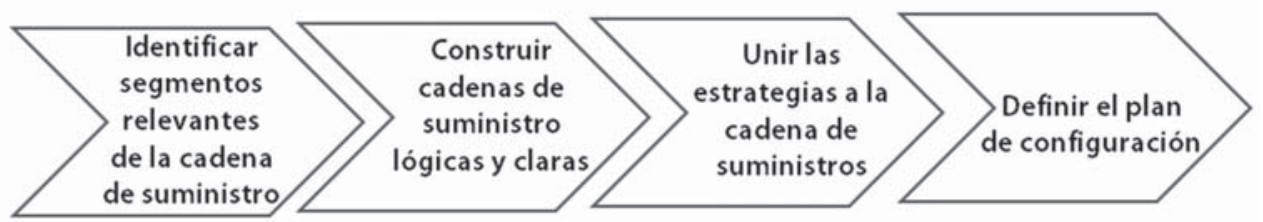

Identificar segmentos Construir cadenas de Unir las estrategias a la Definir el plan de conrelevantes de la cade- suministro lógicas y cadena de suministros figuración na de suministro claras

\begin{tabular}{|c|c|c|c|c|}
\hline Propósito & $\begin{array}{l}\text { Entender las diferen- } \\
\text { cias logísticas de los } \\
\text { clientes y productos. }\end{array}$ & $\begin{array}{l}\text { Definir cadenas de su- } \\
\text { ministro claras basadas } \\
\text { en servir necesidades } \\
\text { únicas de varios seg- } \\
\text { mentos. }\end{array}$ & $\begin{array}{l}\text { Realizar selecciones } \\
\text { estratégicas de acuer- } \\
\text { do con el tipo de ca- } \\
\text { dena de suministro. }\end{array}$ & $\begin{array}{l}\text { Definir la estructura de } \\
\text { las ventajas y procesos } \\
\text { para apoyar la nueva } \\
\text { configuración. }\end{array}$ \\
\hline Acercamiento & $\begin{array}{l}\text { Usar variables relevan- } \\
\text { tes de segmentación } \\
\text { de la cadena de sumi- } \\
\text { nistro; obtener puntos } \\
\text { de vista de los clientes } \\
\text { y socios de la cadena } \\
\text { de suministro. }\end{array}$ & $\begin{array}{l}\text { Encontrar el balance } \\
\text { ente lo real y la escala; } \\
\text { definir enfoques es- } \\
\text { tratégicos para cada } \\
\text { cadena de suministro. }\end{array}$ & $\begin{array}{l}\text { Enfocar sobre argu- } \\
\text { mentos estructurales y } \\
\text { política, no en proceso } \\
\text { o en IT. }\end{array}$ & $\begin{array}{l}\text { Priorizar el cambio } \\
\text { basado en el grado } \\
\text { de incompatibilidad } \\
\text { estratégica y dificultad } \\
\text { de implementación. }\end{array}$ \\
\hline Resultado & $\begin{array}{l}\text { Matriz de segmenta- } \\
\text { ción cliente-Producto }\end{array}$ & $\begin{array}{l}\text { Descripción de enfo- } \\
\text { que estratégico y atri- } \\
\text { butos únicos de cada } \\
\text { cadena de suministro. }\end{array}$ & $\begin{array}{l}\text { Definición de estra- } \\
\text { tegia en cada área de } \\
\text { decisión para cadena } \\
\text { de suministro. }\end{array}$ & $\begin{array}{l}\text { Visión del flujo futuro } \\
\text { de la cadena de su- } \\
\text { ministro, procesos y } \\
\text { economía; plan de mi- } \\
\text { gración. }\end{array}$ \\
\hline
\end{tabular}

Tabla 1. Objetivos del modelo de cadena propuesto

Fuente: Llamas y Tasaico 2005.

Para el desarrollo del software debemos seguir unas fases y establecer cada una con cierto criterio, teniendo en cuenta las restricciones del sistema, los objetivos tanto del sistema como del negocio, así como las limitaciones que esta pueda tener. En una primera fase se deben considerar:

a) Ios requerimientos no funcionales;

b) la lista de riesgos y restricciones; y

c) la arquitectura inicial. 
Se deben definir varias tareas, tales como:

a) Organizar el proyecto, esto incluye definir los costos, el equipo que se necesite, la infraestructura, el involucramiento del personal, la traza del horizonte y del tiempo del proyecto.

b) Definir las medidas de desempeño, definir las actividades para llevar a cabo el proyecto.

c) Crear un plan inicial detallado del proyecto, distribuir el tiempo, las reuniones, los recursos involucrados y el personal que se necesite.

d) Capacitar al equipo para el proyecto; se debe ir informando e instruyendo al usuario que hará uso del sistema de información acerca de cómo se desarrollarán las tareas a partir de la implementación del sistema de información, quéventajas puede obtener, despejar falsas ideas sobre las herramientas tecnológicas y hacerles perder el temor al cambio.

e) Revisar la integridad de la base de datos, ya que esta se convertirá en el activo más preciado para la empresa; la información debe estar bajo ciertos estándares de calidad, debe tener disponibilidad inmediata tanto para el almacenamiento como para las consultas que se puedan formular, normas de seguridad, entre otros.

f) Gestionareinstalarel nuevo hardware; en el camino habránvarios requerimientos quizá equipos nuevos; hay que tener en cuenta los requerimientos mínimos para el funcionamiento del sistema de información que permita el óptimo desarrollo del sistema.

g) Instalar el sistema de información, para lo cual se procede a instalar el nuevo software.

h) Integrar los datos, que consiste en la recopilación de la información necesaria para que el sistema funcione.

i) Ejecutar el sistema, que consiste en ponerlo a prueba. Se ejecutan tareas para medir el cumplimiento de las operaciones solicitadas. En este paso se pueden observar si existen deficiencias en el sistema.

j) Mejoramiento continuo como último paso. Es muy importante y se le puede considerar un ciclo sin fin, pues no tiene final; siempre se debe tener una información de cómo va trabajando el sistema, si es que tiene errores o si se debe cambiar algún proceso, o quizá algunos casos de uso ya no sean útiles. Solo así se garantizará el óptimo funcionamiento del sistema.

Finalmente se describen los procesos para desarrollar el proyecto y se define un horizonte de tiempo como el siguiente: 


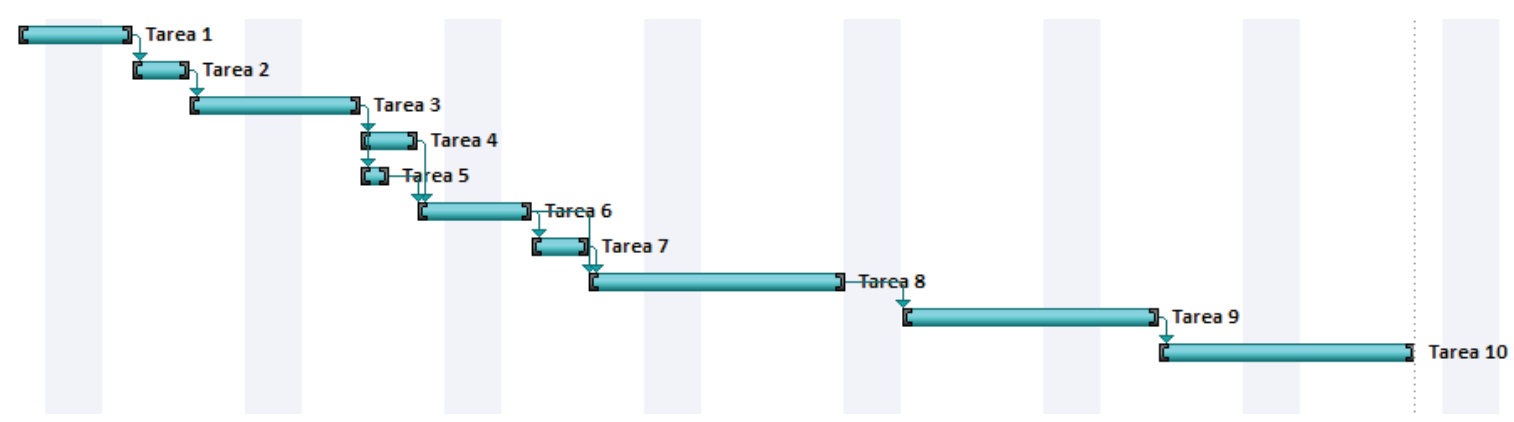

Figura 2. Implementación del proyecto

Elaboración propia.

\begin{tabular}{|l|c|c|c|c|}
\hline Nombre de tarea & Duración & Comienzo & Fin & Costo (S/.) \\
\hline Obtener información de la empresa & 2 días & vie $15 / 03 / 13$ & lun 18/03/13 & 150.0 \\
\hline Obtener procesos de la empresa & 2 días & mar $19 / 03 / 13$ & mié $20 / 03 / 13$ & 250.0 \\
\hline Obtener problemas de los procesos & 4 días & jue $21 / 03 / 13$ & mar 26/03/13 & 3250.0 \\
\hline Modelamiento de procesos & 2 días & mié $27 / 03 / 13$ & jue 28/03/13 & 1300.0 \\
\hline $\begin{array}{l}\text { Identificar requerimientos funcionales } \\
\text { y no funcionales }\end{array}$ & 1 día & lun 01/04/13 & lun 01/04/13 & 1000.0 \\
\hline $\begin{array}{l}\text { Elaborar diseño físico y lógico de la } \\
\text { solución }\end{array}$ & 2 días & mar 02/04/13 & mié 03/04/13 & 550.0 \\
\hline Documentar casos de uso & 2 días & jue 04/04/13 & vie 05/04/13 & 525.0 \\
\hline Elaborar documentación del sistema & 7 días & lun 08/04/13 & mar 16/04/13 & 990.0 \\
\hline Desarrollo del sistema & 120 días & mié 17/04/13 & mar 01/10/13 & 11000.0 \\
\hline Pruebas y aceptación del sistema. & 7 días & mié 02/10/13 & jue 10/10/13 & 1100.0 \\
\hline
\end{tabular}

Tabla 2. Duración y costo del proyecto

Elaboración propia.

40 
El tiempo total del proyecto y su costo total son 151 días y S/. 20.115,00, respectivamente. Para gestionar la toma de decisiones se busca manipular la información por medio de herramientas de Tl; la propuesta de solución brindará los recursos necesarios para transformar los datos en indicadores entendibles, los cuales servirán para la toma de decisiones, para ver el cumplimiento de los objetivos y para monitorear los procesos según su desempeño, para lo cual se busca implementar una solución BI.

Se deben tener en cuenta los objetivos principales, de donde se desprenderán los requerimientos y funcionalidades que conduzcan a la solución; para determinar el problema los indicadores más claros son los pronósticos, la rentabilidad y las operaciones.

Las estadísticas juegan un papel importante, ya que con estas se podrá validar la solución propuesta; también se incluyen herramientas de minería de datos. Existen diversos software estadísticos, los más usados son Minitab y MS Excel; para minería de datos, Weka; mientras que para Bl existe Microsoft Business Intelligence, Qlikview. Para el desarrollo se tienen que considerar ciertas actividades:

a) Planificación del proyecto, que consiste básicamente en definir el proyecto y su alcance.

b) Definición de los requerimientos: el detalle de la solución y las métricas necesarias solicitadas por el usuario.

c) Diseño de la arquitectura.

d) Manipulación de los datos: creación del ETL, transformación de la información.

e) Implementación del proyecto, es decir, el desarrollo de la solución.

f) Mantenimiento del proyecto.

g) Mejora continua.

\section{Metodología de solución}

\subsection{Modelamiento de procesos}

\subsubsection{Inventario}

El proceso de inventario será soportado por el sistema de información, con lo cual se busca mejorar la gestión del inventario, teniendo en cuenta los incumplimientos que se puedan presentar. Además, se genera un pronóstico de la demanda y un plan de suministros, que se sustentan con la información que se recopila de las actividades de la empresa y las actividades básicas de este proceso que se siguen desarrollando. 


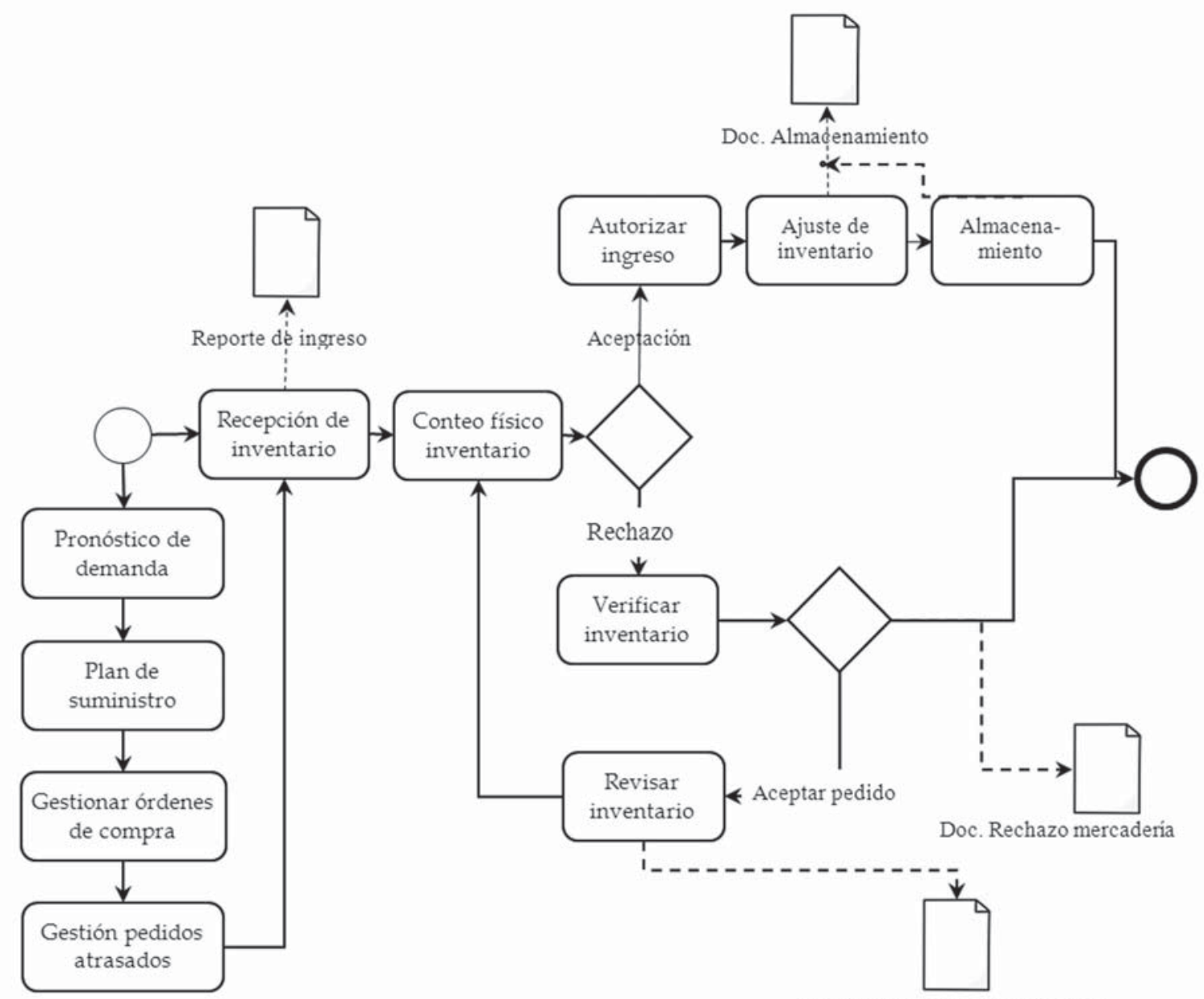

Doc. Observación de mercaderia

Figura 3. Proceso de inventario

Elaboración propia. 


\subsection{Ventas}

El proceso de ventas sigue el mismo camino pues es soportado por el plan de suministros, el pronóstico de la demanda y las ventas; los procesos básicos son soportados por el sistema de información, con lo cual se va recopilando información para ir desarrollando el plan de suministros.

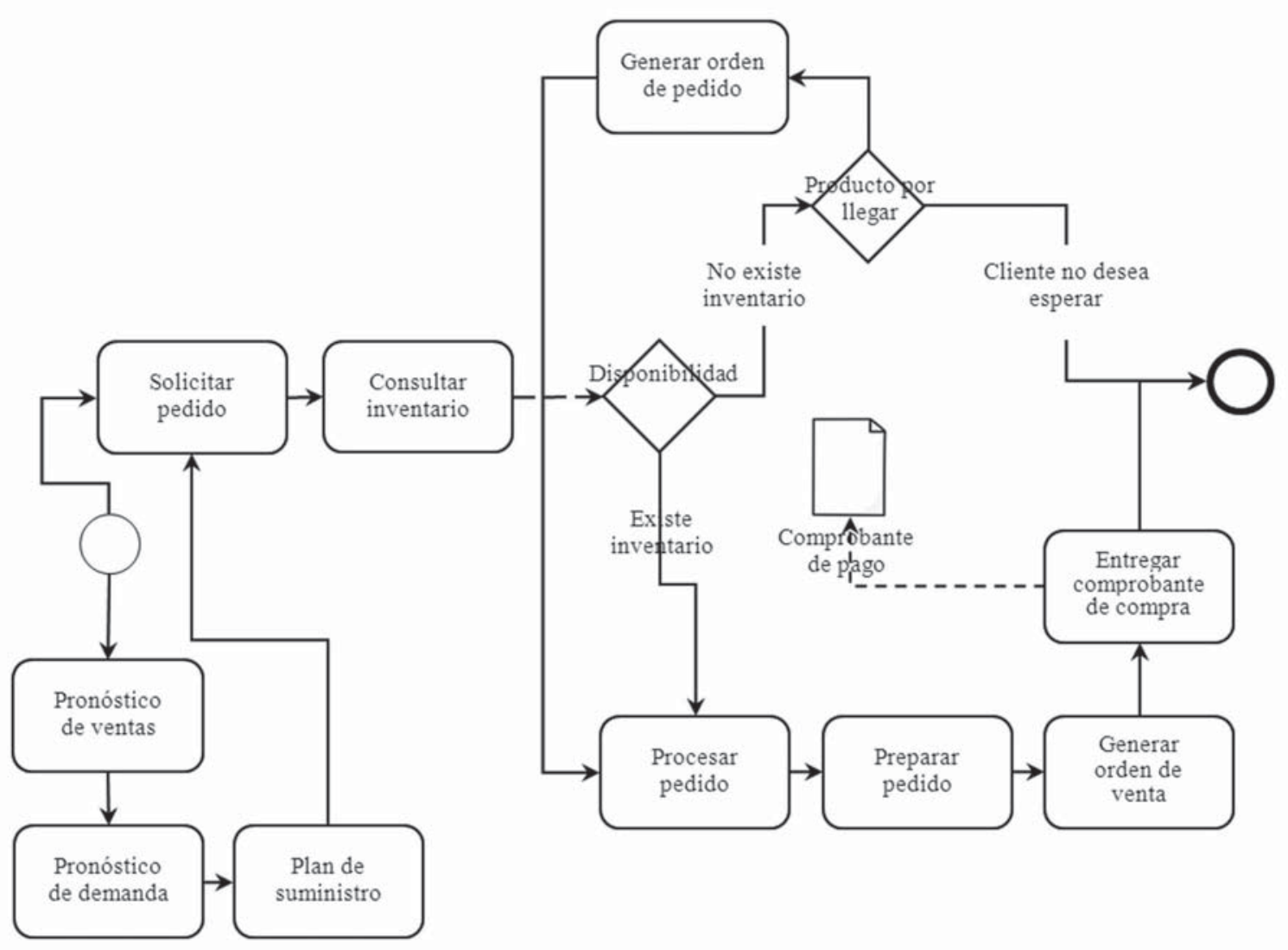

Figura 4. Proceso de ventas

Elaboración propia. 


\subsection{Simulación de procesos}

Para el problema planteado se propone un modelo de cadena de suministros simulado simulado por medio de la dinámica de sistemas, para lo cual se utilizará el software PowerSim.

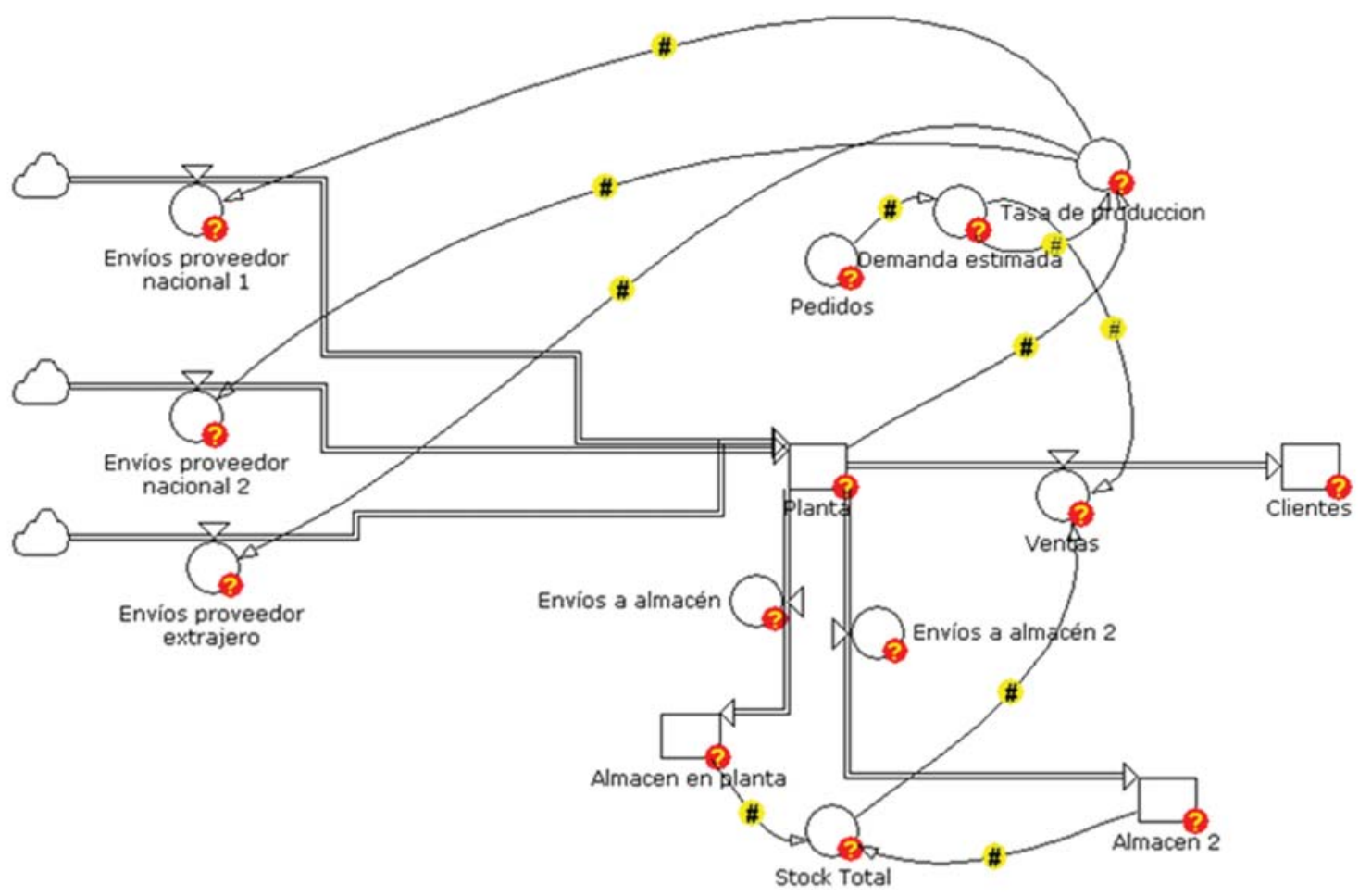

Figura 5. Modelo de cadena de suministros propuesto

Elaboración propia.

En el figura 5 se puede identificar la lista de proveedores; se toman en cuenta tres proveedores, dos nacionales y uno extranjero, por el hecho de que ellos representan entre $80 \%$ y $90 \%$ de los ingresos de materia prima. Estas pasan a la planta principal, la cual contiene un almacén; la empresa cuenta con otro almacén externo; las ventas se realizan de manera directa en planta y el modelo puede cambiar mientras va evolucionando la cadena.

Las áreas que abarcará el sistema de información son las de ventas e inventario, así como el área administrativa. Por temas de gestión y manejo de información, 
el usuario final tiene una interfaz con la cual va a interactuar; la aplicación es soportada por un servidor de datos (BD) con la información histórica ya almacenada y la información actual, que el propio sistema otorgará. La información ya almacenada servirá para crear reportes de ventas, inventario y el cruce entre estos, para así obtener información más detallada. Por último, están los recursos que son básicamente las herramientas que complementan el sistema. Se requiere un motor de base de datos, el aplicativo y el sistema que gestione la conexión.

Los equipos utilizados serán un servidor central, un router/switch para realizar la conexión entre el servidor y los equipos, y tener escalabilidad en el sistema. Se contará con cuatro áreas: ventas, producción, inventario y administración; la conexión será por medio de cable UTP, pero a su vez se busca tener conexión inalámbrica para tener el manejo del sistema en cualquier ubicación así como otros dispositivos de acceso a futuro.

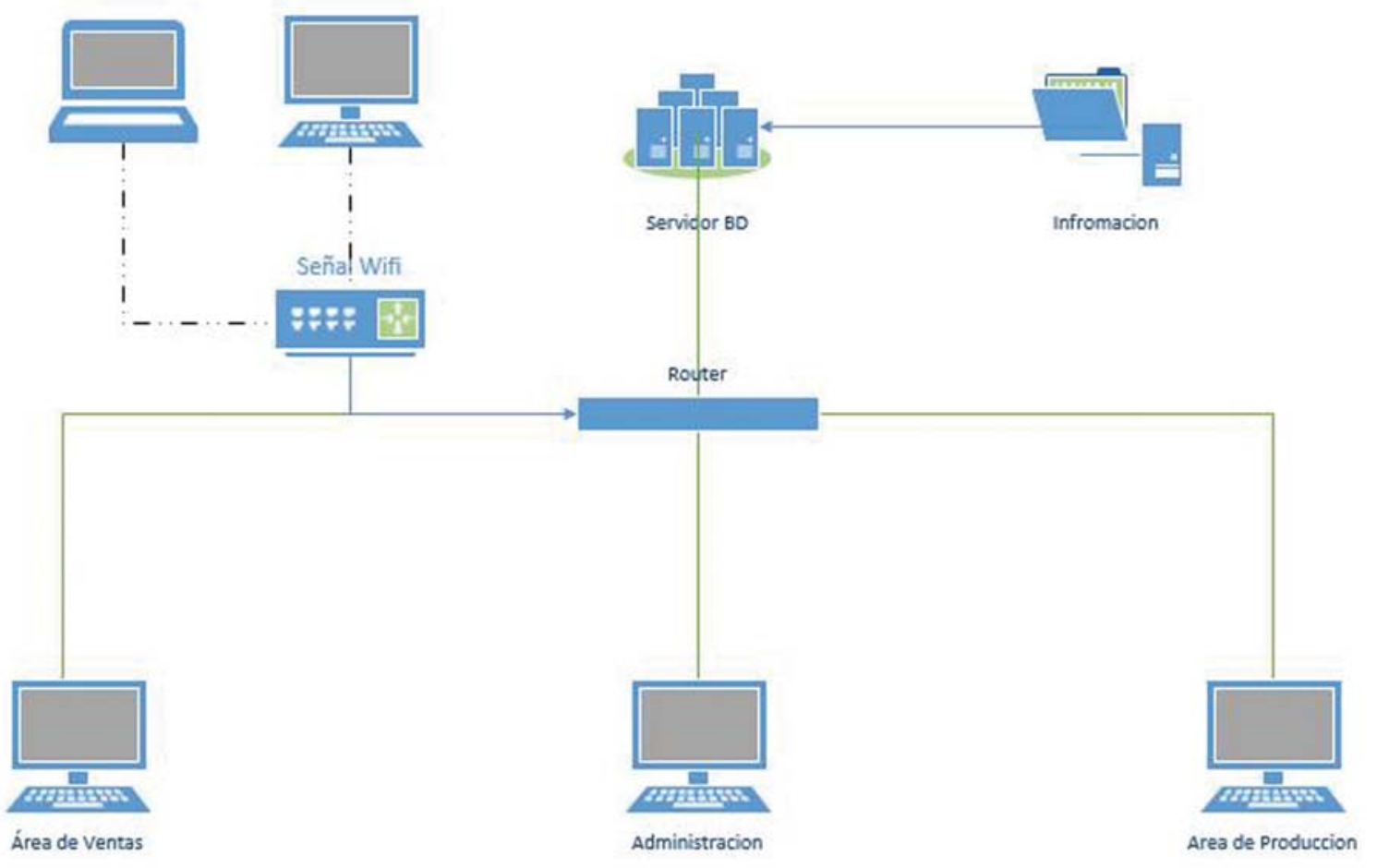

Elaboración propia.

Figura 6. Diseño físico del sistema

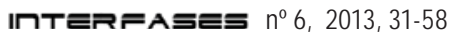


Se consideran las áreas y procesos que están involucrados en el sistema de información. Es necesario automatizar los procesos de ventas, inventario, producción y administración. Se cuenta con el modelo de paquetes y las funciones que cada uno posee.

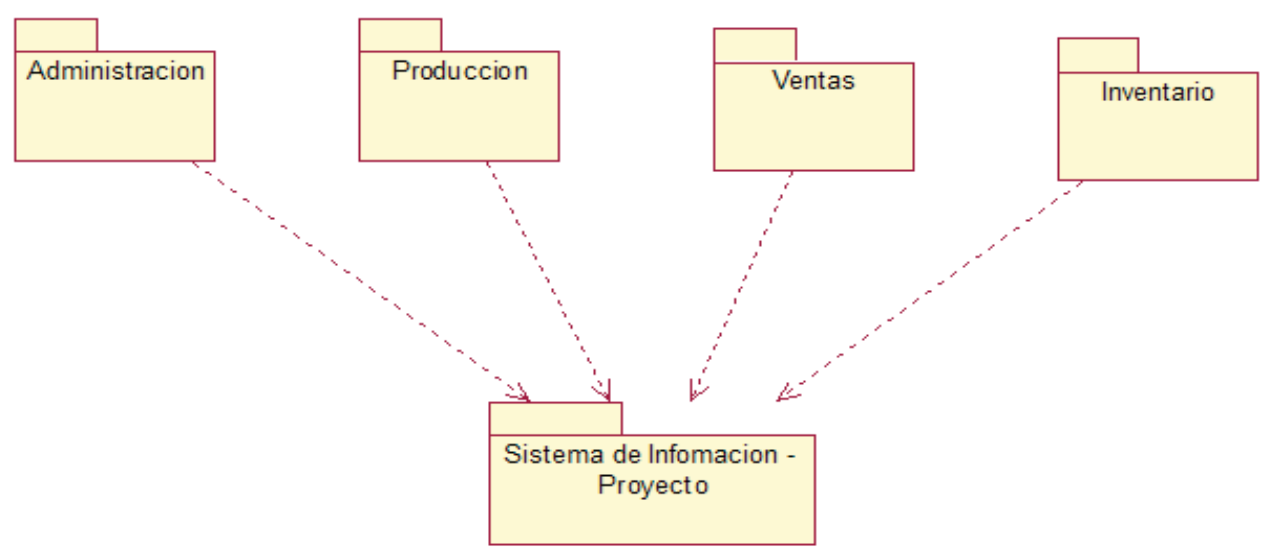

Figura 7. Diagrama de paquete

Fuente: Elaboración propia.

Se identifican los procesos, casos de uso de los actores del negocio.

\subsection{Requerimientos}

\subsubsection{Requerimientos no funcionales}

Se procede a describir los requerimientos no funcionales según las necesidades del usuario y del sistema.

a) Portabilidad.- el sistema de información debe ser capaz de reutilizarse en vez de crearse un nuevo código cuando el software pasa de una plataforma a otra.

b) Flexibilidad.- el sistema de ventas debe ser flexible a los cambios que se requieran realizar; los productos están en constantes cambios y se necesita que el sistema se adapte a estos. 
Cómo elevar la rentabilidad de una empresa. Aplicación de un ERP nacional

c) Rendimiento/tiempo de respuesta.- la aplicación debe proporcionar un rendimiento de 2 segundos de tiempo de respuesta para el $90 \%$ de las solicitudes.

d) Seguridad.- todos los accesos deben ser autenticados y autorizados utilizando un protocolo seguro para que la información sea verídica.

e) Usabilidad.- el componente de la interfaz debe poder ejecutarse bajo cualquier tipo de circunstancia.

f) Disponibilidad.- el sistema debe estar apto para funcionar las 24 horas de los 7 días de la semana para los 365 días del año, con una disponibilidad total de $99 \%$.

\subsubsection{Requerimientos funcionales}

\section{a) Casos de uso administración}

- Administrar usuario: eliminar usuario, modificar usuario, registrar usuario.

- Gestionar reportes.

- Administrar comprobantes: crear comprobante, modificar/eliminar comprobante.

- Administrar proveedores: registrar proveedores, modificar/eliminar proveedores.

- Administrar producto: registrar producto, registrar categoría de producto, asignar inventario, revisar almacén, administrar inventario. 


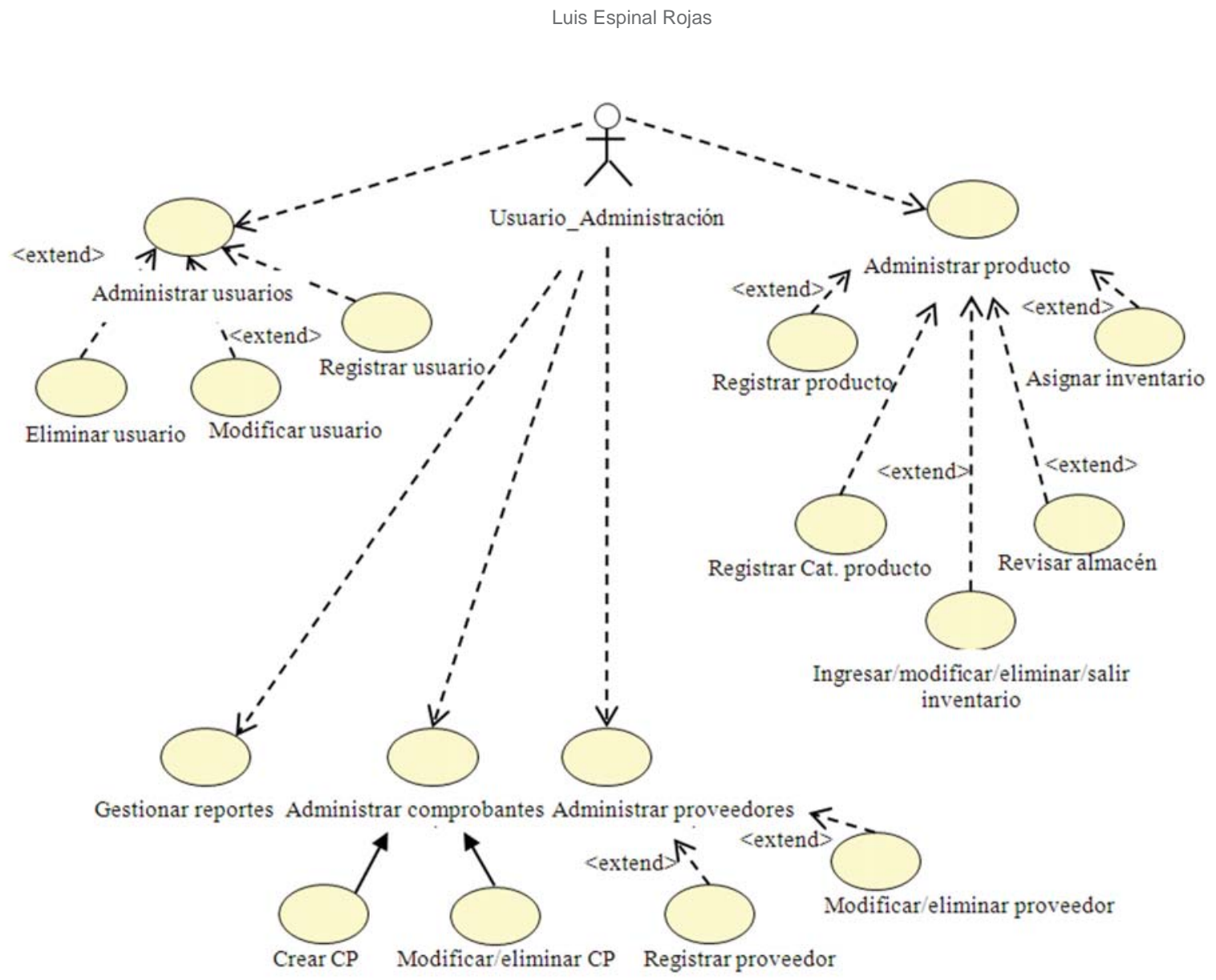

Figura 8. Casos de uso administración

Fuente: Elaboración propia. 
b) Diagrama de componentes

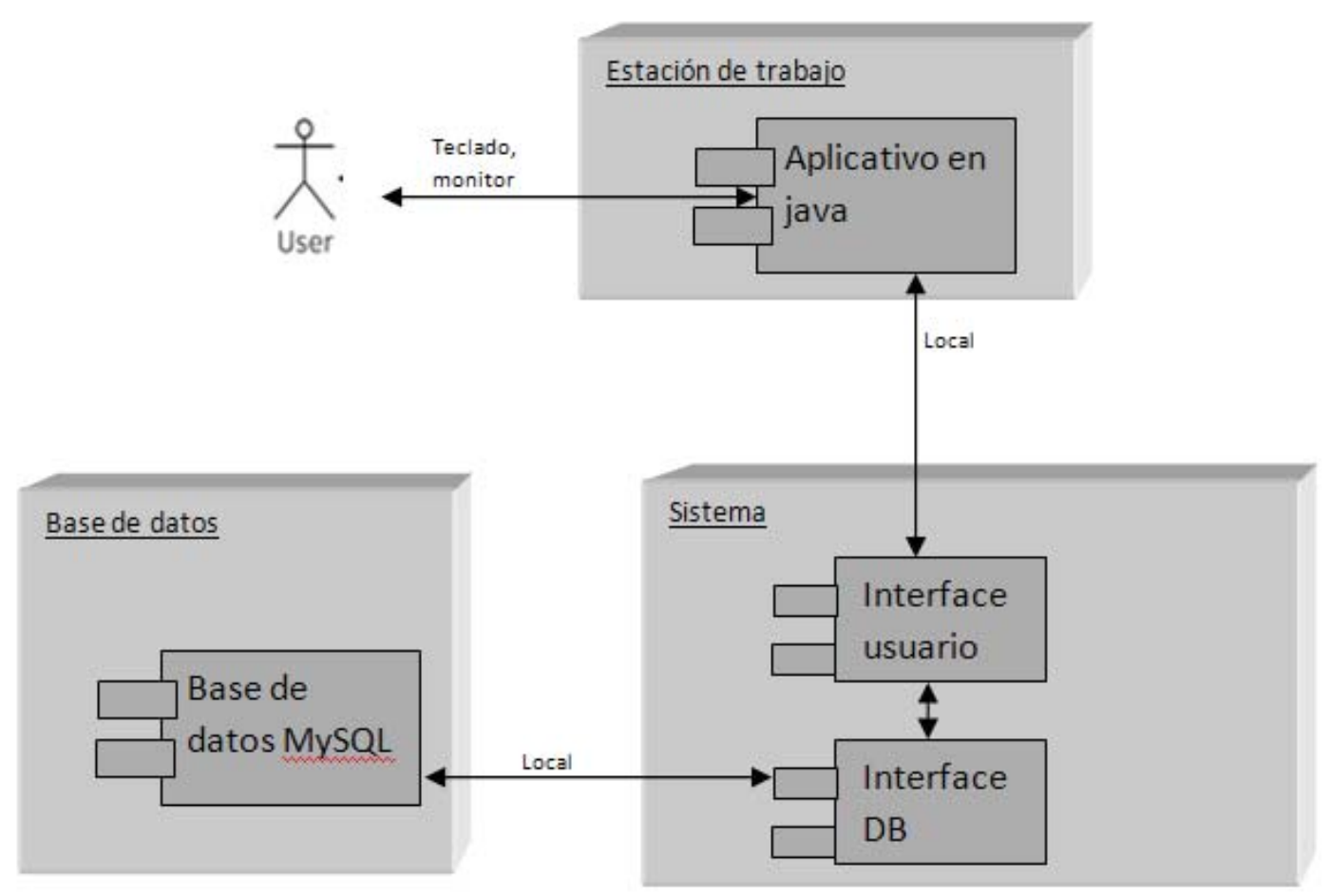

Figura 9. Diagrama de componentes

Fuente: Elaboración propia.

\section{c) Tablas de datos}

El usuario ingresará a través del ejecutable y tendrá acceso a la interfaz de inicio por medio local; de igual manera, a la base de datos utilizando el motor de base datos, MySQL. Se obtiene así el diagrama de la base de datos implementados, en la cual se identifican las siguientes tablas:
a) tipo_documento;
b) cliente;
c) producto;
d) categoria;
e) contado; 

f) venta;
g) operacion;
h) personal_si;
i) compra_crédito.
j) compra_contado.
k) usuario;
l) cronograma_pago; y
m) proveedor.

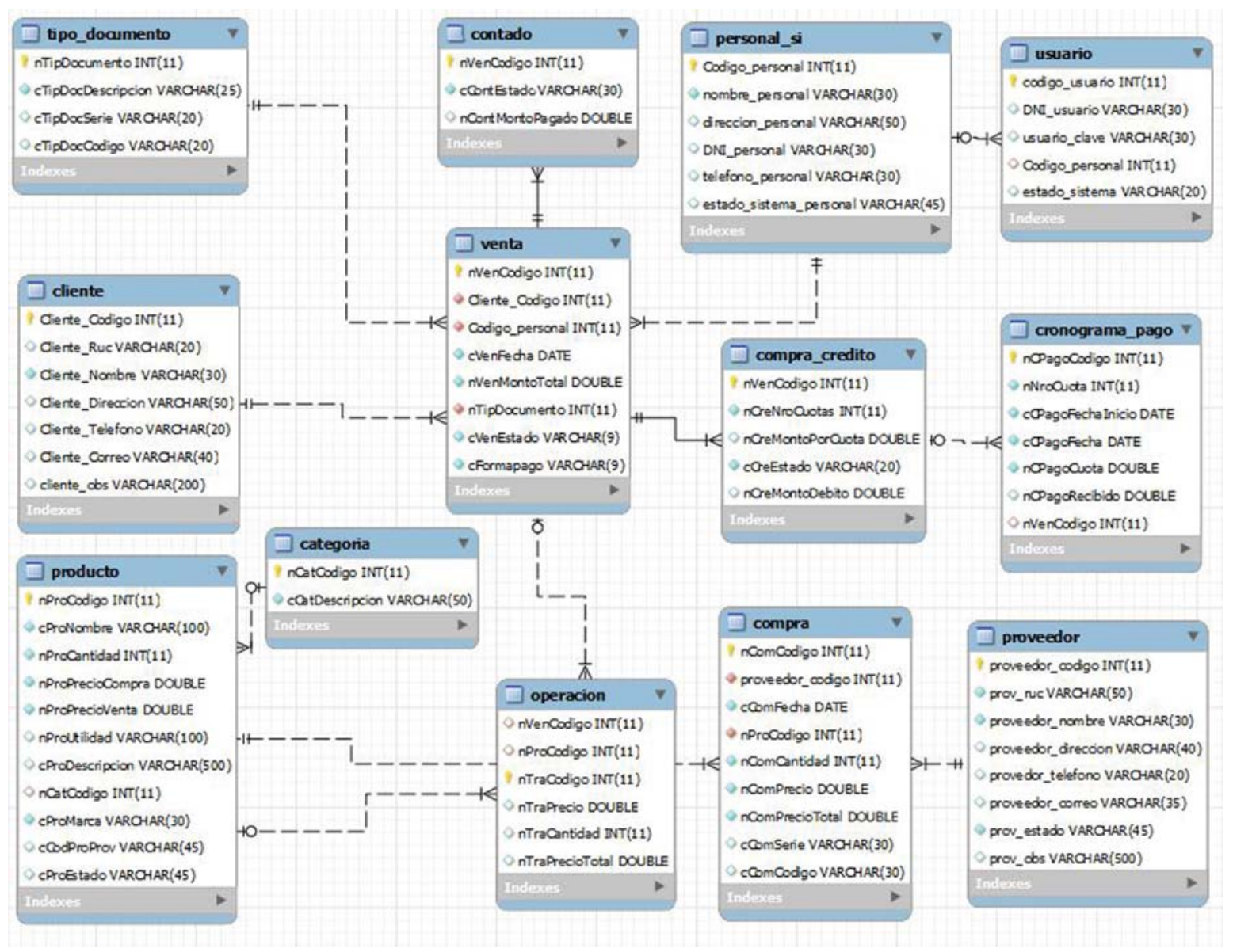

Figura 10. Diagrama de base de datos

Elaboración propia. 
Se diseñan los reportes que va a generar el sistema.

\begin{tabular}{|c|c|c|c|c|c|}
\hline & & & & & IECHA: new DateO \\
\hline & \multicolumn{4}{|c|}{ LISTA DE PRODUCTOS } & \multirow[b]{2}{*}{ PRECIO UNTT. } \\
\hline NOMERE & DESCRIRCION & MARCA & CATEGORIA & STOCK & \\
\hline SI \{NOMBRE $\}$ & $\$$ F $\{$ DESCRIPCION\} & SF $\{$ MARCA\} & II \{CATEGORIA $\}$ & \$F $\{$ STOCK $\}$ & \$F Bs. \\
\hline \multicolumn{6}{|c|}{ "Pagina" + SV\{PAGE_NUMBER\}+" de""'" + SV\{PAGE_NUMBER\} } \\
\hline
\end{tabular}

Figura 11. Modelo de reporte

Elaboración propia.

Para el sistema Bl se establecen indicadores y con los datos recolectados se efectúa el desarrollo del tablero de indicadores. Se procede a describir cada parte del tablero de indicadores implementados. El usuario puede seleccionar el mes al que desea acceder, la categoría de producto o el año, según sus necesidades. En la figura 12 se puede apreciar el porcentaje de ventas de los productos según su categoría, asimismo el mes y año que el usuario ha seleccionado anteriormente.

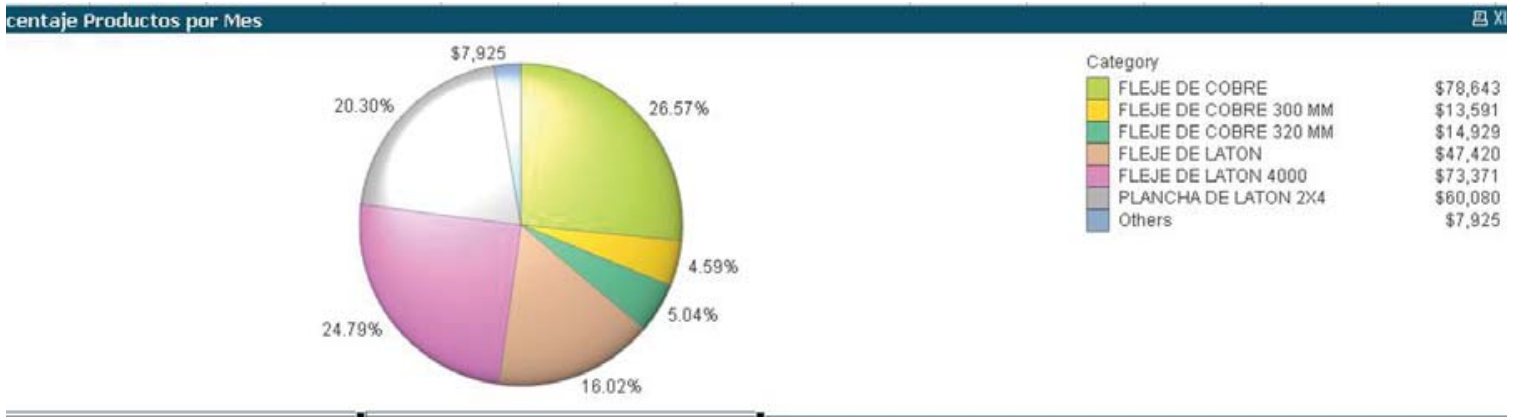

Figura 12. Nivel de ventas por mes

Elaboración propia.

\section{Impacto financiero}

Para la verificación y validación del sistema se ejecutarán las siguientes tareas con el fin de obtener pronósticos de la demanda, a ser comparados con las ventas reales y cuantificar las posibles pérdidas en las que puede estar incurriendo la empresa. 
a) Elaboración de la lista de productos.

b) Recolección de las ventas por cada producto por un periodo de 54 meses.

c) Reconocer productos representativos.

d) Elaboración del gráfico de ventas.

e) Elaborar pronósticos de demanda utilizando metodología Arima.

f) Recolección ventas año 2013.

g) Comparación y cuantificación de datos históricos.

El nivel de ventas actualizado antes del inicio del proyecto se presenta en la tabla 3; el proyecto salió a prueba en el mes de septiembre del año 2013.

\begin{tabular}{|c|c|c|}
\hline Año & Ventas (\$) & $\begin{array}{c}\text { Variación } \\
\text { porcentual }\end{array}$ \\
\hline 2008 & 1250000.0 & - \\
\hline 2009 & 1256321.0 & 0,51 \\
\hline 2010 & 1336895.0 & 6,41 \\
\hline 2011 & 1426111.0 & 6,67 \\
\hline 2012 & 1475965.0 & 3,50 \\
\hline 2013 & 1489652.0 & 0,93 \\
\hline Total & $\$ 8234944.0$ & \\
\hline
\end{tabular}

Tabla 3. Nivel de ventas actualizado al tercer trimestre del 2013

Elaboración propia.

El nivel de ventas en el último trimestre del año 2013 ha aumentado significativamente en comparación con el trimestre pasado, lo que lleva a concluir que las ventas anuales tendrán un crecimiento de más del $20 \%$. En la tabla 4 se comparan los datos estimados: 
Cómo elevar la rentabilidad de una empresa. Aplicación de un ERP nacional

\begin{tabular}{|c|c|c|c|c|c|c|c|c|c|c|c|}
\hline \multirow{2}{*}{ Descripción } & \multirow{2}{*}{ Unidad } & \multicolumn{2}{|c|}{ Set. } & \multicolumn{2}{|c|}{ Oct. } & \multicolumn{2}{|c|}{ Nov. } & \multicolumn{2}{|c|}{ Dic. } & \multicolumn{2}{|c|}{ Ene. } \\
\hline & & $\mathbf{P}$ & V & $\mathbf{P}$ & V & $\mathbf{P}$ & V & $\mathbf{P}$ & V & $\mathbf{P}$ & v \\
\hline $\begin{array}{l}\text { Plancha de } \\
\text { latón 1/16" 2x4 } \\
\text { Import. }\end{array}$ & Unidad & 50 & 30 & 37 & 66 & 54 & 42 & 38 & 41 & 61 & 39 \\
\hline $\begin{array}{l}\text { Plancha de } \\
\text { latón 1/40" } 2 \times 4 \\
\text { Import. }\end{array}$ & Unidad & 180 & 140 & 101 & 79 & 116 & 72 & 97 & 85 & 139 & 101 \\
\hline $\begin{array}{l}\text { Plancha de } \\
\text { latón 1/54" } 2 \times 4 \\
\text { Import. }\end{array}$ & Unidad & 137 & 181 & 170 & 82 & 141 & 141 & 143 & 143 & 145 & 151 \\
\hline $\begin{array}{l}\text { Plancha de latón } \\
1 \mathrm{~mm} \times 400 \mathrm{~mm} \\
\text { Import. }\end{array}$ & $\mathrm{kg}$ & 71.67 & 89.63 & 101.1 & 86.28 & 101.12 & 141.16 & 414.85 & 400.85 & 87.18 & 65.18 \\
\hline $\begin{array}{l}\text { Plancha de latón } \\
2.5 \mathrm{~mm} \times 320 \\
\mathrm{~mm} \mathrm{~S} / \mathrm{D}\end{array}$ & $\mathrm{kg}$ & 100.69 & 2.69 & 87.7 & 3.2 & 2.75 & 2.77 & 2.76 & 2.87 & 2.77 & 1.79 \\
\hline P: Pronóstico, V: & tas & & & & & & & & & & \\
\hline
\end{tabular}

Tabla 4. Comparación de datos estimados

Fuente: Elaboración propia.

Se realiza la comparación solo en cinco productos de la empresa y se observa una mejora en el nivel de ventas; si se cuantifican los ingresos, el ingreso adicional solo en estos productos sería de US\$28366.43, tal como se aprecia en la Tabla 5.

\begin{tabular}{|l|c|c|c|c|c|c|c|c|}
\hline Descripción & Unidad & $\begin{array}{l}\text { Precio } \\
\text { venta } \\
\text { (US\$) }\end{array}$ & Set & Oct & Nov & Dic & Ene & $\begin{array}{c}\text { Total } \\
\text { (US\$) }\end{array}$ \\
\hline Plancha de latón 1/16" $2 \times 4$ import. & Unidad & 171.43 & 20 & & 14 & & 22 & 9257.14 \\
\hline Plancha de latón 1/40" $2 \times 4$ import. & Unidad & 73.21 & 40 & 22 & 44 & 12 & 38 & 11421.43 \\
\hline Plancha de latón 1/54" $2 \times 4$ import. & Unidad & 55.36 & & 88 & & & 4871.43 \\
\hline $\begin{array}{l}\text { Plancha de latón 1mm x } 400 \mathrm{~mm} \\
\text { import. }\end{array}$ & $\mathrm{kg}$ & 12.86 & & 14.82 & & 14 & 22 & 653.40 \\
\hline $\begin{array}{l}\text { Plancha de latón } 2.5 \mathrm{~mm} \times 320 \mathrm{~mm} \\
\text { S/D }\end{array}$ & $\mathrm{kg}$ & 11.79 & 98 & 84.55 & & & 0.98 & 2163.03 \\
\hline
\end{tabular}

Elaboración propia.

Tabla 5. Cuantificación de ingresos adicionales 
La variación comparada con el trimestre anterior es amplia, con lo que se cumpliría el objetivo al finalizar el periodo, superando drásticamente las ventas del año 2013. En la tabla 6 se muestran los ingresos por ventas al cerrar el año 2013, y se aprecia que la variación de las ventas supera los pronósticos.

\begin{tabular}{|c|c|c|}
\hline Año & Ventas (US\$) & $\begin{array}{c}\text { Variación } \\
\text { porcentual }\end{array}$ \\
\hline 2008 & 1250000.0 & - \\
\hline 2009 & 1256321.0 & 0.51 \\
\hline 2010 & 1336895.0 & 6.41 \\
\hline 2011 & 1426111.0 & 6.67 \\
\hline 2012 & 1475965.0 & 3.50 \\
\hline 2013 & 1795652.0 & 21.66 \\
\hline Total & 8540944.0 & \multicolumn{2}{|l}{} \\
\hline
\end{tabular}

Tabla 6. Nivel de ventas actualizado a fines del 2013.

Elaboración propia.

\section{Conclusiones}

Se puede observar de manera general que todas las empresas independientemente del rubro en el que estén deben tener un control del inventario que les permita su trazabilidad. Lo que se busca es tener un control del inventario perpetuo y, con ello, tener la cantidad necesaria de inventario en el momento y el lugar deseados y en las condiciones óptimas. Se pueden apreciar los siguientes beneficios inmediatos al disponer de una buena gestión del inventario:

a) Mejoramiento de la rentabilidad de la empresa.- se aumentó en más del $20 \%$, en comparación con el año anterior, aplicando la nueva metodología de trabajo solo en un último trimestre del último año.

b) Mejoramiento de la imagen de la empresa.- los pedidos son atendidos de manera eficiente.

c) Menos reproceso.- se redujo el costo de operaciones ya que se eliminaron los procesos innecesarios.

d) Disminución de los costos de manejo de inventario.- el nivel de inventario disminuyó, lo que permite un mejor control del costo que estos implican. 
e) Reducción de sobrecostos.- al tener poco inventario se puede reducir el margen de variación de precio de los productos.

f) Entrega de pedidos a tiempo.- el área de producción y ventas posee un mejor control de los inventarios asignados, lo que permite tener siempre disponible el producto solicitado.

Los beneficios se pueden cuantificar demostrando numéricamente la necesidad de la solución propuesta. Para el caso desarrollado, se han identificado varios problemas en el manejo del inventario, los cuales fueron atacados uno por uno para así tener una mejor gestión de los procesos en la empresa:

a) Manejo de inventario ineficiente.

b) Políticas de trabajo que no están definidas.

c) Procesos de trabajo ineficientes.

d) Los pedidos son entregados fuera de fecha.

e) No se maneja inventario de seguridad.

f) No existe un plan de demanda que gestione los pedidos con anticipación para evitar roturas de stock.

g) Se incurre en sobrecostos de inventario.

h) Niveles de stock no tienen control o plan de mitigación.

En el trabajo descrito se pudo acceder a los datos de las ventas de la empresa, los cuales fueron analizados para revisar la variación que presentan a lo largo del tiempo. Se analizaron las variaciones y se escogieron cinco productos representativos por el comportamiento que estos presentan en variabilidad, estacionalidad y frecuencia. Los mismos datos fueron analizados por la metodología de trabajo Arima, con los que se determinaron los pronósticos para el último trimestre del año.

Se realizó la comparación de los pronósticos y las ventas reales, se pudieron apreciar las diferencias y se averiguó si en esa fecha la empresa presentó algún problema con el inventario, lo que llevó a revisar una lista de incidencias en las que se puede apreciar que su principal problemas es la falta de inventario.

El proyecto afectó positivamente el nivel de las ventas en el último trimestre del año; solo en ese periodo de tiempo las ventas variaron positivamente; se puede apreciar que pasó de un crecimiento anual moderado que va de un 2,34 \% en promedio, media geométrica, a un incremento de $21,66 \%$ en el último año, lo que hizo cumplir con la meta en un corto periodo de tiempo, y si nos ponemos a analizar el nivel de ventas en el último trimestre se puede apreciar que la empresa tuvo ingresos de US\$28.366,43 tan solo por cinco productos; cabe resaltar que la empresa cuenta con más de 50 productos, entre materia prima que se vende y productos que se fabrican. El resultado permite estimar cuánto más puede crecer 
la empresa en el presente año si esta ya cuenta con el apoyo del sistema, además de ver nuevas formas de cómo mejorar los procesos, aplicar nuevas herramientas de Tl e introducir nuevos sistemas.

\section{Bibliografía}

Althonayan, M. y A. Papazafeiropoulou (2013). Evaluating the performance on ERP systems in king saud university (ksu): a stakeholders' perspective. 2013 46th Hawaii International Conference on System Sciences. Volumen 0. Los Alamitos, CA: IEEE Computer Society, doi:10.1109/HICSS.2013.210.

Au, K. F. y D. C. K. Ho (2002). "Electronic commerce and supply chain managment: Value-adding service for clothing manufacturers". Integrated Manufacturing Systems 13(4)

Ben-Menachem, M. y G. S. Marliss (2004). "Inventorying information technology systems: supporting the paradigm of change". IEEE Software 21(5).

Chen, Y. et al. (2011). The causes and prevention measures of bullwhip effect in supply chain. International Conference on Information Management, Innovation Management and Industrial Engineering. Volumen 1. Los Alamitos, CA: IEEE Computer Society, doi:10.1109/ICIII.2011.113.

Cuéllar, Guillermo. Metodología para la implementación de sistemas ERP. <http:// fccea.unicauca.edu.co/old/erp.htm>. [Consulta: 9 de noviembre del 2012].

Dixit, A. K. y Prakash, O. (2011). "A study of issues affecting ERP implementation in SMEs". Researchers World 2(2).

Espinal, A. A. C.; Montoya, R. A. G. y J. A. C. Arenas (2010). Gestión de almacenes y tecnologías de la información y comunicación. Estudios Gerenciales 26(117).

Fayyad, U.; Piatetsky-Shapiro, G. y P. Smyth (1996). "From data mining to knowledge discovery in databases".

Gestión y Administración de Empresas (s.f.). <http://www.gestionyadministracion. com/empresas/herramientas-de-gestion.htm>. [Consulta: 2 de noviembre del 2012].

Izquierdo, L. et al. (11 de noviembre del 2008). Modelado de sistemas complejos mediante simulación basada en agentes y mediante dinámica de sistemas. $<$ http://www.luis.izqui.org/papers/Izquierdo_Galan_Santos_Olmo_2008. pdf $>$. [Consulta: 10 de mayo del 2013].

"Las herramientas de gestión a favor de la empresa moderna (n.d.). <http://www. articuloz.com/administracion-articulos/las-herramientas-de-gestion-afavor-de-la-empresa-moderna-726394.html>. [Consulta: 7 de julio del 2013]. 
Laukkanen, S.; Sarpola, S., y P. Hallikainen (2007). "Enterprise size matters: objectives and constraints of ERP adoption". Journal of Enterprise Information Management, 20(3), doi:10.1108/17410390710740763.

Llamas, Daniel y Delia Tasaico (2005). "Análisis y modelamiento de la cadena de suministro del gas natural en el Perú y su impacto ambiental". Investigación del Instituto de Investigación Científica de la Universidad de Lima.

Lu, Y. y T. Kakola (2013). An information system design product theory for integrated order, transportation and warehouse management systems. 2013 46th Hawaii International Conference on System Sciences. Volumen 0. Los Alamitos, CA: IEEE Computer Society, doi:10.1109/HICSS.2013.87.

Marbán, Ó.; Mariscal, G., y J. Segovia (2009). A data mining \& knowledge discovery process model.

McConnell, J., Nunnally, B. y B. McGarvey (2012). "Process modeling: a powerful weapon in the fight to reduce variation-part 1". Journal of Validation Technology 18(2).

Noguera, Manuel (2011). Introducción al modelado de procesos de negocio $<$ http://www.ugr.es/ mnoguera/collaborative_systems-business_ processes_10-11.pdf >. [Consulta: 8 de noviembre del 2012].

Othman, S. N., y N. H. Mustaffa (2012). Supply chain simulation and optimization methods: an overview. Intelligent Systems, Modelling and Simulation, International Conference. Volumen 0. Alamitos, CA: IEEE Computer Society, doi:10.1109/ISMS.2012.122.

Pearson, M. (2012). "Strategies for aligning manufacturing with business and supply chain goals". Logistics Management 51 (2).

Qiongwei, Y.; Guangxing, S. y L. Zhendong (2010). An empirical study of business intelligence (bi) application in e-business enterprises: taking ynyy pharmaceutical chain enterprise as example. Management of e-Commerce and e-Government, International Conference. Volumen 0. Los Alamitos, CA: IEEE Computer Society, doi:10.1109//CMeCG.2010.70.

Rivera, E. (2008). Asesoría Informática. <http://www.asesoriainformatica.com/ erp_01.htm>. [Consulta: 2 de noviembre del 2012].

Sánchez, V. (27 de agosto del 2007). "Planea los recursos de tu pyme". Reforma. $<$ http://search.proquest.com/docview/307880085?accountid=45277>. México. [Consulta: 5 de mayo del 2007].

Sun, C. y Zhang G. (2012). An empirical investigation of the impact of scm components on the logistics service performance. In International Symposium on Distributed Computing and Applications to Business, Engineering and 
Science. Volumen 0. Los Alamitos, CA: IEEE Computer Society, doi:10.1109/ DCABES.2012.120.

Tsai, W.-C. y A.-P. Chen (2008). Global asset allocation using xcs experts in countryspecific ETFs. Convergence Information Technology, International Conference. Volumen 2. Los Alamitos, CA: IEEE Computer Society, doi:10.1109/ICCIT.2008.418.

Urdaneta, E. (30 de abril del 2013). <http://www.alejandrosueldo.com.ar/>. Obtenido de Bl \& TI Inteligencia de Negocios y Tecnología: <http:// www.alejandrosueldo.com.ar/joomla15/index.php?option=com_conte $n t \& v i e w=$ article\&id $=37:$ fundamentos-del-data-mining\&catid $=15$ : datawarehousing\&ltemid $=2>$.

Valencia, V. (2 de mayo del 2013). "Logran ventajas implementando ERP". El Norte. <http://search.proquest.com/docview/312143096?accountid=45277>. Monterrey.

Valencia, V. (8 de marzo del 2004). "Ofrecen ERP escalable para PyMES". El Norte. Monterrey.

Wenrich, K. I.y N. Ahmad (2009). "Lessons learned during a decade of ERP experience: a case study". International Journal of Enterprise Information Systems 5(1).

Williams, B. D., y T. Tokar, (2008). A review of inventory management research in major logistics journals: Themes and future directions. International Journal of Logistics Management. 19(2), doi:10.1108/09574090810895960.

Xiaoping, Q. et al. (2009). The development of inventory management information system based on workflow technology. Electronic Commerce and Security, International Symposium. Volumen 1. Los Alamitos, CA: IEEE Computer Society, doi:10.1109/ISECS.2009.255

Zhou, L. (2012). Research on the integration application of business intelligence and ERP in management of e-commerce and e-government. International Conference. Volumen 0. Los Alamitos, CA: IEEE Computer Society, doi:10.1109/ ICMeCG.2012.42

Zhou, L. (2011). The inventory problem of the supply chain management: how to form a win-win among the supply chain stakeholders. 2012 Second International Conference on Business Computing and Global Informatization. Volumen 0. Los Alamitos, CA: IEEE Computer Society, doi:10.1109/BCGIn.2011.31 